\title{
Switching vaccination schemes for epidemic models with distributed time delay and two types of noise
}

\section{X.Y. Wang ${ }^{1 *}$ (D)}

"Correspondence:

wangxiying668@163.com ${ }^{1}$ College of Science, Henan University of Technology, Zhengzhou, 450001, China

\section{Springer}

\begin{abstract}
Novel switched HIV/AIDS (human immunodeficiency virus/acquired immune deficiency syndrome) epidemic models with distributed time delay and bounded noise and Gaussian white noise are developed and investigated using stochastic Itô's lemma and the Lyapunov-Razumikhin method. New criteria depending on these factors are established to confirm that the disease-free equilibrium of the model is stochastically asymptotically stable as the threshold parameter is less than unity, which implies that the disease eventually disappears theoretically. Otherwise, the disease persists weakly. Further, the main results show that the threshold values are related to two types of noise and time delay. Pulse control strategies are then applied to two types of the infected population, the susceptible population, and the infected population, respectively. More precisely, the effects of each control strategy on the stochastic solution of the model are evaluated to justify the relation between control parameters and threshold parameters of the model. In comparison with the basic reproduction number of the model with pulse control, it is easily found that the main results in these references are improved and extended. Finally, four examples are presented to support the main results, and one future research direction is suggested.
\end{abstract}

Keywords: Switched epidemic model; Distributed time delay; Bounded noise and Gaussian white noise; Stochastic asymptotic stability; Lyapunov-Razumikhin method; Pulse vaccination strategies

\section{Introduction}

It is well known that the HIV/AIDS epidemic is still one of the major global public health issues. It is reported by the World Health Organization that millions of people die of the AIDS disease every year. Most people are at higher risk of acquiring HIV infection when they keep sexual contacts with the AIDS patients [1]. Thus, it is an extremely complex issue to prevent or control AIDS effectively.

In epidemic dynamics modeling, mathematical models play a significant role in research into spreading and controlling the disease [2-6]. In particular, since the initial HIV/AIDS models were proposed by May and Anderson [7], many various refinements have been added to modify these models [8-10]. For instance, Saha et al. [11] modeled a compartment HIV/AIDS model including treatment and pre-exposure prophylaxis, and analyzed

(c) The Author(s) 2021. This article is licensed under a Creative Commons Attribution 4.0 International License, which permits use sharing, adaptation, distribution and reproduction in any medium or format, as long as you give appropriate credit to the original author(s) and the source, provide a link to the Creative Commons licence, and indicate if changes were made. The images or other third party material in this article are included in the article's Creative Commons licence, unless indicated otherwise in a credit line to the material. If material is not included in the article's Creative Commons licence and your intended use is not permitted by statutory regulation or exceeds the permitted use, you will need to obtain permission directly from the copyright holder. To view a copy of this licence, visit http://creativecommons.org/licenses/by/4.0/. 
local and global stability of equilibrium points. Hsieh et al. [12] developed new HIV/AIDS models with commercial sex workers and sexually active male customers, and discussed the global asymptotic stability of the disease-free equilibrium. Naresh et al. [13] incorporated both horizontal and vertical transmission into an AIDS epidemic model and studied the dynamics of the model. By establishing the optimal control equations, condoms and treatment regime have an important influence on the dynamics of HIV/AIDS models [14].

Traditionally, infectious disease model coefficients are assumed to be constant in time. However, a more realistic approach is to suppose that these parameters are time-varying. This is because the alteration of environment often leads to great changes in the host population behavior, which causes the model parameters no longer to be constant. For instance, researchers $[15,16]$ assumed that the epidemic model parameters could be abruptly varying in time and developed epidemic models with the term-time forcing. Besides, Liu and Wang [17] incorporated switching into epidemic models and investigated their global stability. These models may be called switched systems. One main feature of a switched system with unstable subsystems is that the system may be stable due to the included switching rule impact $[18,19]$. We will utilize this technique to investigate the dynamics of epidemic models.

Moreover, in some practical models, their development trend is relevant to not only current but past state. This phenomenon is usually called delay. Population dynamics is inevitably influenced by time delay. The emergence of time delay could affect the stability of equilibria, and delay systems exhibit more complicated dynamics behavior such as oscillation, bifurcation, and other phenomena [20-23]. For instance, Cai et al. [24] introduced a discrete time delay to an HIV/AIDS model and discussed the effect of the time delay on the stability of the endemically infected equilibrium. Bera et al. [25] studied the dynamics of a delay HIV infection model and described the influence of the delay on the stability of the model. Muhammad et al. [26] considered a delay model for immune system-tumor interaction and analyzed the stability of the tumor-free steady state and the tumor-persistent steady state. To the best of our knowledge, there have been few works done to study the global asymptotic stability of switched HIV/AIDS epidemic models with delay. Therefore, it is urgent to incorporate delay parameters and switching parameters into HIV/AIDS epidemic models and investigate their dynamics.

On the other hand, the environmental factors and the immunological state of the host have great influence on population dynamics, which causes that the disease-free equilibrium or endemic equilibrium is not a fixed status. Recently, stochastic epidemic models have received increasing attention [27-29]. In stochastic AIDS models, noise is assumed to be Gaussian noise [30]. However, in epidemic models, different populations vary enormously in terms of growth and decay processes. Thus, it is very necessary to introduce the combined Gaussian white noise and bounded noise into the HIV/AIDS models by assuming that models' parameters are replaced by different random excitation in this paper. Furthermore, some diseases (for instance, small pox and measles) can be prevented or contained by pulse vaccination. Thus, pulse vaccination is becoming a significant issue in the study of epidemic models [31]. Samanta et al. [32] considered a stochastic chlamydia epidemic model with pulse vaccination strategy. They found that the disease could be eradicated when impulse vaccination rate is larger than some critical value. The research results show that pulse vaccination strategy with high values of vaccination has gained 
more prominence than conventional continuous vaccination [33]. There has been a focus in the literature on studying pulse vaccination epidemic models [34].

Motivated by the above discussion, we aim in this paper to formulate and investigate new HIV/AIDS models including switching parameters, distributed time delay, and two types of noise. More specifically, stability results ensuring the disease eradication or persistence under new threshold conditions are developed by using stochastic Itô's lemma and the Lyapunov-Razumikhin method. Pulse vaccination strategies are applied to two types of the infected population, the susceptible population, and the infected population, respectively. Compared with the existing models without pulse vaccination strategy, our proposed models take into account the efficiency of vaccine and are more useful in eradicating the disease. Therefore, this paper will improve the existing HIV/AIDS models and will further extend current knowledge on HIV/AIDS model methods.

This paper is organized as follows. In Sect. 2, a new switched HIV/AIDS epidemic model with two types of noise and distributed time delay is formulated. Stability theorems for the disease extinction or persistence are given in Sect. 3. In Sect. 4, pulse vaccination schemes are added into the above model. Threshold criteria involving pulse parameters are derived by redefining similar basic reproduction numbers. Some examples are presented to demonstrate the obtained results in Sect. 5.

\section{Formulation of the model}

In this section, we develop a switched HIV/AIDS epidemic model with distributed time delay and bounded noise and Gaussian white noise. The total number of high-risk individuals $N(t)$ at time $t$ are partitioned into the following compartments: the susceptible individuals $S(t)$ who are uninfected, the asymptomatic infected individuals $I_{1}(t)$ who have been infected but have no symptoms of the disease, the symptomatic infected individuals $I_{2}(t)$ who have been infected and have symptoms of the disease, and the full AIDS individuals $A(t)$, i.e., $N(t)=S(t)+I_{1}(t)+I_{2}(t)+A(t)$.

Moreover, the vital dynamic and epidemiological assumptions are made as follows [3537]. Since different individuals experience different growth and decay processes, assume that bounded noise and Gaussian white noise are incorporated into an HIV/AIDS model based on the parameter perturbation method. Suppose that the incubation time is a distributed parameter over the interval $[0, \tau]$, in which $\tau>0$ is called an upper bound of the incubation time in the infective individuals. Assume that the nonnegative and continuous function $f$ is a distribution function of incubation times and satisfies the following: (1) $f:[0, \tau) \rightarrow[0, \infty)$ is nondecreasing; (2) $\int_{0}^{\tau} f(h) d h=1, \int_{0}^{\tau} h f(h) d h<\infty$. Noting that the spread of AIDS changes over time, the model parameters are assumed to be switching parameters which could switch their functional forms. Suppose that a switching signal $\sigma(t)$ controls these switching parameters and satisfies the following conditions: (1) It is a piecewise continuous (from the left) function; (2) $\sigma(t):\left(t_{k-1}, t_{k}\right] \rightarrow\{1,2, \ldots, m\}, k=1,2, \ldots$, in which $m$ is the number of subsystems, $t_{k}>t_{k-1}$, and $t_{k} \rightarrow \infty$ as $k \rightarrow \infty$. The set of all switching rules is represented by $\mathcal{I}$.

In this paper, $\lambda$ denotes a recruitment rate of the susceptible individuals from the larger embedding population. The switched parameter $p_{\sigma}$ is a transmission rate between the susceptible individuals and the asymptomatic infected individuals, and the force of infection is $p_{\sigma} S(t) \int_{0}^{\tau} I_{1}(t-h) d h$; The switched parameter $q_{\sigma}$ is a transmission rate between the susceptible individuals and the symptomatic infected individuals, and the force of infection is $q_{\sigma} S(t) \int_{0}^{\tau} I_{2}(t-h) d h$. Denote the natural death ratio of all human classes by $a$. 
The asymptomatic infected individuals become the symptomatic infected individuals at a rate of $e_{\sigma} r_{\sigma}$ by a screening method. The asymptomatic infected individuals become the full blown AIDS individuals at a rate of $\left(1-e_{\sigma}\right) r_{\sigma} ; \rho_{\sigma}^{1}$ denotes a disease-caused death ratio of the asymptomatic infected individuals; $b_{\sigma}$ is a disease-caused death rate of the symptomatic infected individuals; and $c_{\sigma}$ is a disease-caused death ratio of the full blown AIDS individuals. The symptomatic infected individuals become the full blown AIDS individuals at a rate of $\rho_{\sigma}^{2}$.

Under the above assumptions, a switched HIV/AIDS epidemic model with distributed time delay and bounded noise and Gaussian white noise is presented as follows:

$$
\left\{\begin{aligned}
d S(t)= & \left\{\lambda-p_{\sigma} S(t) \int_{0}^{\tau} I_{1}(t-h) d h-q_{\sigma} S(t) \int_{0}^{\tau} I_{2}(t-h) d h-a S(t)\right\} d t \\
& -S(t) d M(t), \\
d I_{1}(t)= & \left\{p_{\sigma} S(t) \int_{0}^{\tau} I_{1}(t-h) d h+q_{\sigma} S(t) \int_{0}^{\tau} I_{2}(t-h) d h-\left(r_{\sigma}+a+\rho_{\sigma}^{1}\right) I_{1}(t)\right\} d t \\
& -\varphi_{1} I_{1}(t) d W_{1}(t), \\
d I_{2}(t)= & \left\{e_{\sigma} r_{\sigma} I_{1}(t)-\left(a+b_{\sigma}+\rho_{\sigma}^{2}\right) I_{2}(t)\right\} d t-\varphi_{2} I_{2}(t) d W_{2}(t), \\
d A(t)= & \left\{\left(1-e_{\sigma}\right) r_{\sigma} I_{1}(t)+\rho_{\sigma}^{2} I_{2}(t)-c_{\sigma} A(t)-a A(t)\right\} d t-\varphi_{3} A(t) d W_{3}(t),
\end{aligned}\right.
$$

in which the initial conditions satisfy

$$
S\left(t_{0}\right)>0, \quad I_{1}\left(t_{0}\right) \geq 0, \quad I_{2}\left(t_{0}\right) \geq 0, \quad A\left(t_{0}\right) \geq 0 .
$$

From physical considerations, all quantities $\lambda, p_{\sigma}, q_{\sigma}, a, r_{\sigma}, \rho_{\sigma}^{1}, e_{\sigma}, b_{\sigma}, \rho_{\sigma}^{2}$, and $c_{\sigma}$ are positive and continuous functions of time for $\sigma \in\{1,2, \ldots, m\}$.

In system (1), bounded noise $\frac{d M(t)}{d t}$ is of the form $\frac{d M(t)}{d t}=\Lambda \sin \left(\Omega t+\varphi W_{0}(t)+U\right)$, in which $\Lambda, \Omega$, and $\varphi$ are positive constants; $\Lambda$ is an amplitude of bounded noise and $\Omega$ is its center frequency; $U$ is distributed in $[0,2 \pi]$ as a random phase uniformly. The standard Brownian motions $W_{0}(t), W_{1}(t), W_{2}(t)$, and $W_{3}(t)$ are defined on the probability space $(\Omega, \mathcal{F}, \mathcal{P})$, in which $\left\{\mathcal{F}_{t}\right\}_{t \geq 0}$ satisfies the following conditions: (1) It is increasing; (2) It is right-continuous as $\mathcal{F}_{0}$ contains all $\mathcal{P}$-null sets. Moreover, $\eta_{i}=\frac{d W_{i}(t)}{d t}(i=0,1,2,3)$ is independent Gaussian white noise with the intensity of noise $\varphi_{i}(i=0,1,2,3)$ characterized by

$$
\left\langle\eta_{i}(t)\right\rangle=0, \quad\left\langle\eta_{i}\left(t_{1}\right) \eta_{i}\left(t_{2}\right)\right\rangle=\delta\left(t_{1}-t_{2}\right), \quad \text { and } \quad\left\langle\eta_{i}\left(t_{1}\right) \eta_{j}\left(t_{2}\right)\right\rangle=0 \quad(i \neq j)
$$

in which $\langle\cdot\rangle$ denotes the average over the ensemble of the stochastic process, and $\delta(t)$ is the dirac delta function. Note that $Q_{0}=(\lambda / a, 0,0,0,0)$ is a disease-free equilibrium of system (1) without noise compartments. Since system (1) is affected by a fluctuating environment, it is necessary to investigate that $Q_{0}$ is stochastically asymptotically stable to exhibit whether the disease dies out or not.

As the expected number of secondary cases produced by a typical infective individual in entirely susceptible population, the basic reproduction number can be computed by defining the spectral radius of the matrix $F V^{-1}[38,39]$. In the following section, we derive the basic reproduction number of system (1) and then investigate stochastic asymptotic stability of the system. 


\section{Global dynamics of system (1)}

In this section, we first extend some notations and propositions of system (1) and then investigate its global asymptotic stability.

Definition $1([40])$ The population $Z(t)$ will go to extinction with probability 1 if $\lim \sup _{t \rightarrow \infty} Z(t)=0$.

Definition 2 ([40]) The population $Z(t)$ is weakly persistent with probability 1 if $\lim \sup _{t \rightarrow \infty} Z(t)>0$.

Proposition 1 If $\left(T\left(t_{0}\right), I_{1}\left(t_{0}\right), I_{2}\left(t_{0}\right), A\left(t_{0}\right)\right) \in R_{+}^{4}$ is any initial value of system (1), then there exists a unique solution $\left(T(t), I_{1}(t), I_{2}(t), A(t)\right)$ on $t \geq t_{0}$ and the solution will remain in $R_{+}^{4}$ with probability 1 , where $R_{+}^{4}=\left\{x \in R_{+}^{4} \mid x_{i}>0, i=1,2,3,4\right\}$.

Proof The proof is similar to Dalal et al. [27] and hence is omitted.

Proposition 2 Assume that $a>\Lambda$. The solution $\left(T(t), I_{1}(t), I_{2}(t), A(t)\right)$ of system (1) with initial conditions (2) satisfies

$$
\limsup _{t \rightarrow \infty} N(t) \leq \frac{\lambda}{\Delta} \quad \text { with probability } 1,
$$

in which $N(t)=S(t)+I_{1}(t)+I_{2}(t)+A(t)$ and $\Delta=\min _{\sigma \in\{1,2, \ldots, m\}}\left\{a-\Lambda, a+\rho_{\sigma}^{1}, a+b_{\sigma}, a+c_{\sigma}\right\}$.

Proof By Proposition 1, we can compute the derivative of $N(t)$ along system (1) as follows:

$$
\begin{aligned}
d N(t)= & d S(t)+d I_{1}(t)+d I_{2}(t)+d A(t) \\
\leq & \left\{\lambda-(a+\Lambda) S(t)-\left(a+\rho_{\sigma}^{1}\right) I_{1}(t)-\left(a+b_{\sigma}\right) I_{2}(t)-\left(c_{\sigma}+a\right) A(t)\right\} d t \\
& -\left\{\varphi_{1} I_{1}(t) d W_{1}(t)+\varphi_{2} I_{2}(t) d W_{2}(t)+\varphi_{3} A(t) d W_{3}(t)\right\} .
\end{aligned}
$$

We take the expectation of $N(t)$ for Eq. (4), then

$$
\limsup _{t \rightarrow \infty} N(t) \leq \frac{\lambda}{\Delta} \quad \text { with probability } 1,
$$

in which $\Delta=\min _{\sigma \in\{1,2, \ldots, m\}}\left\{a-\Lambda, a+\rho_{\sigma}^{1}, a+b_{\sigma}, a+c_{\sigma}\right\}$. This completes the proof of Proposition 2.

Denote $p^{1}=\min _{\sigma \in\{1,2, \ldots, m\}}\left\{p_{\sigma}\right\}$, and $q^{1}=\min _{\sigma \in\{1,2, \ldots, m\}}\left\{q_{\sigma}\right\}$. Then we have the following proposition.

Proposition 3 Assume that $a>\Lambda$. For any $\epsilon>0$, the solution $\left(T(t), I_{1}(t), I_{2}(t), A(t)\right)$ of system (1) with initial conditions (2) with probability 1 satisfies

$$
G=\frac{\lambda}{\left(p^{1}+q^{1}\right) \frac{\lambda}{\Delta} \tau+a+\Lambda} \leq \liminf _{t \rightarrow \infty} S(t) \leq \limsup _{t \rightarrow \infty} S(t) \leq \frac{\lambda}{a-\Lambda},
$$

where $\Delta$ is given in Proposition 2. 
Proof From the first equation of system(1), we have

$$
\begin{aligned}
d S(t)= & \left\{\lambda-p_{\sigma} S(t) \int_{0}^{\tau} I_{1}(t-h) d h-q_{\sigma} S(t) \int_{0}^{\tau} I_{2}(t-h) d h-a S(t)\right\} d t \\
& -S(t) d M(t) \\
\leq & \lambda-(a-\Lambda) S(t) d t .
\end{aligned}
$$

It follows that $\lim \sup _{t \rightarrow \infty} S(t) \leq \frac{\lambda}{a-\Lambda}$.

On the other hand, from Proposition 2 , for any $\epsilon>0$, there exist large sufficient $t_{1}>0$ and $t \geq t_{1}$ such that $I_{1}(t) \leq \frac{\lambda}{\Delta}+\epsilon$ and $I_{2}(t) \leq \frac{\lambda}{\Delta}+\epsilon$. Hence, based on the first equation of system(1), we obtain that

$$
d S(t) \geq\left\{\lambda-\left[\left(p^{1}+q^{1}\right)\left(\frac{\lambda}{\Delta}+\epsilon\right) \tau+a+\Lambda\right] S(t)\right\} d t
$$

It follows that $\liminf _{t \rightarrow \infty} S(t) \geq \frac{\lambda}{\left(p^{1}+q^{1}\right)\left(\frac{\lambda}{\Delta}+\epsilon\right) \tau+a+\Lambda}$. Since $\epsilon$ can be sufficiently small, the result is valid. This completes the proof.

In the following, an approximate basic reproduction number of system (1) is presented, and then it is shown that the disease will be cleared out with probability 1 when the approximate basic reproduction number is less than one; the disease will persist weakly with probability 1 when the approximate basic reproduction number is greater than one.

Theorem 1 Assume that $\left(T(t), I_{1}(t), I_{2}(t), A(t)\right)$ is any solution of system $(1)$ with the initial value $\left(T\left(t_{0}\right), I_{1}\left(t_{0}\right), I_{2}\left(t_{0}\right), A\left(t_{0}\right)\right) \in \mathbb{R}_{+}^{4}$, and $a>\Lambda$. Assume that

$$
\bar{R}_{0}=\sup _{t \geq l} \frac{1}{t} \int_{t_{0}}^{t} \frac{\lambda \tau q_{\sigma} B_{\sigma}+e_{\sigma} r_{\sigma}(a-\Lambda) D_{\sigma}}{a-\Lambda} d s
$$

for some $l \geq t_{0}, B_{\sigma}=\frac{a-\Lambda}{\lambda \tau\left(q_{\sigma}-p_{\sigma}\right)+(a-\Lambda)\left(r_{\sigma}+a+\rho_{\sigma}^{1}\right)}>0$, and $D_{\sigma}=\frac{1}{e_{\sigma} r_{\sigma}+a-b_{\sigma}-\rho_{\sigma}^{2}}>0$. If $\bar{R}_{0}<1$, then $Q_{0}=(\lambda / a, 0,0,0,0)$ is stochastically asymptotically stable, which implies that the disease in system (1) will go to extinction with probability 1.

Proof Define the set of Lyapunov functions:

$$
\begin{aligned}
V_{\sigma}= & B_{\sigma} I_{1}(t)+D_{\sigma} I_{2}(t)+B_{\sigma} p_{\sigma} \int_{0}^{\tau} \int_{t-h}^{t} S(u+h) I_{1}(u) d u d h \\
& +B_{\sigma} q_{\sigma} \int_{0}^{\tau} \int_{t-h}^{t} S(u+h) I_{2}(u) d u d h,
\end{aligned}
$$

where $B_{\sigma}$ and $D_{\sigma}$ are defined in the theorem. 
According to Proposition 3 and taking the derivative of along system (1), we obtain the following expression:

$$
\begin{aligned}
d V_{\sigma}= & B_{\sigma} d I_{1}(t)+D_{\sigma} d I_{2}(t)+B_{\sigma} p_{\sigma} \int_{0}^{\tau}\left\{S(t+h) I_{1}(t)-S(t) I_{1}(t-h)\right\} d h d t \\
& +B_{\sigma} q_{\sigma} \int_{0}^{\tau}\left\{S(t+h) I_{2}(t)-S(t) I_{2}(t-h)\right\} d h d t \\
= & B_{\sigma}\left\{-\left(r_{\sigma}+a+\rho_{\sigma}^{1}\right) I_{1}(t)+p_{\sigma} \int_{0}^{\tau} S(t+h) I_{1}(t) d h\right. \\
& \left.+q_{\sigma} \int_{0}^{\tau} S(t+h) I_{2}(t) d h\right\} d t+D_{\sigma}\left\{e_{\sigma} r_{\sigma} I_{1}(t)-\left(a+b_{\sigma}+\rho_{\sigma}^{2}\right) I_{2}(t)\right\} d t \\
& -B_{\sigma} \varphi_{1} I_{1}(t) d W_{1}(t)-D_{\sigma} \varphi_{2} I_{2}(t) d W_{2}(t) \\
\leq & \left\{B_{\sigma}\left(p_{\sigma} \frac{\lambda \tau}{a-\Lambda}-r_{\sigma}-a-\rho_{\sigma}^{1}\right) I_{1}(t)+D_{\sigma} e_{\sigma} r_{\sigma}\right\} I_{1}(t) d t \\
& +\left\{B_{\sigma} q_{\sigma} \frac{\lambda \tau}{a-\Lambda}-D_{\sigma}\left(a+b_{\sigma}+\rho_{\sigma}^{2}\right) I_{2}(t)\right\} d t \\
& -B_{\sigma} \varphi_{1} I_{1}(t) d W_{1}(t)-D_{\sigma} \varphi_{2} I_{2}(t) d W_{2}(t) \\
\leq & \left(R_{\sigma}-1\right)\left(I_{1}(t)+I_{2}(t)\right) d t-B_{\sigma} \varphi_{1} I_{1}(t) d W_{1}(t)-D_{\sigma} \varphi_{2} I_{2}(t) d W_{2}(t),
\end{aligned}
$$

in which $R_{\sigma}=\frac{\lambda \tau q_{\sigma} B_{\sigma}+e_{\sigma} r_{\sigma}(a-\Lambda) D_{\sigma}}{a-\Lambda}$.

Then integrating the above inequality over $\left[t_{k-1}, t_{k}\right]$, we have

$$
\begin{aligned}
V_{\sigma}(t) \leq & V_{\sigma}\left(t_{k-1}\right)+\int_{t_{k-1}}^{t}\left\{\left(R_{\sigma}(s)-1\right) I_{1}(s) d s-B_{\sigma}(s) \varphi_{1} I_{1}(s) d W_{1}(s)\right\} \\
& +\int_{t_{k-1}}^{t}\left\{\left(R_{\sigma}(s)-1\right) I_{2}(s) d s-D_{\sigma} \varphi_{2} I_{2}(s) d W_{2}(t)\right\} .
\end{aligned}
$$

Taking the expectation for Eq. (12), we have that

$$
\begin{aligned}
E\left[V_{\sigma}(t)\right] \leq & E\left[V_{\sigma}\left(t_{k-1}\right)\right]+E\left[\int_{t_{k-1}}^{t}\left\{\left(R_{\sigma}(s)-1\right) I_{1}(s) d s-B_{\sigma}(s) \varphi_{1} I_{1}(s) d W_{1}(s)\right\}\right] \\
& +E\left[\int_{t_{k-1}}^{t}\left\{\left(R_{\sigma}(s)-1\right) I_{2}(s) d s-D_{\sigma} \varphi_{2} I_{2}(s) d W_{2}(t)\right\}\right] \\
\leq & E\left[V_{\sigma}\left(t_{k-1}\right)\right]+\int_{t_{k-1}}^{t}\left(R_{\sigma}(s)-1\right) E\left[I_{1}(s)+I_{2}(s)\right] d s .
\end{aligned}
$$

Thus, it can be calculated

$$
E\left[\frac{d V_{\sigma}}{d t}\right] \leq\left(R_{\sigma}-1\right) E\left[I_{1}(t)+I_{2}(t)\right]
$$

Letting $\theta=\min _{\sigma \in\{1,2, \ldots, m\}}\left\{B_{\sigma}, D_{\sigma}\right\}$, it follows that

$$
\theta \frac{d}{d t} E\left[I_{1}(t)+I_{2}(t)\right] \leq E\left[\frac{d V_{\sigma}}{d t}\right] \leq\left(R_{\sigma}-1\right) E\left[I_{1}(t)+I_{2}(t)\right],
$$


i.e.,

$$
\theta \frac{d}{d t} E\left[I_{1}(t)+I_{2}(t)\right] \leq\left(R_{\sigma}-1\right) E\left[I_{1}(t)+I_{2}(t)\right]
$$

For $t \in\left(t_{k-1}, t_{k}\right]$, we obtain that $E\left[I_{1}(t)+I_{2}(t)\right] \leq \frac{1}{\theta} E\left[I_{1}\left(t_{k-1}\right)+I_{2}\left(t_{k-1}\right)\right] \exp \left[\int_{t_{k-1}}^{t}\left(R_{\sigma}(s)-\right.\right.$ 1) $d s]$. Then it follows that, for $t \in\left(0, t_{1}\right], E\left[I_{1}(t)+I_{2}(t)\right] \leq \frac{1}{\theta}\left(I_{1}\left(t_{0}\right)+I_{2}\left(t_{0}\right)\right) \exp \left[\int_{t_{0}}^{t}\left(R_{\sigma}(s)-\right.\right.$ 1)ds]. Similarly, it can be shown that $t \in\left(t_{1}, t_{2}\right], E\left[I_{1}(t)+I_{2}(t)\right] \leq \frac{1}{\theta}\left(I_{1}\left(t_{0}\right)+I_{2}\left(t_{0}\right)\right) \times$ $\exp \left[\int_{t_{0}}^{t_{1}}\left(R_{\sigma}(s)-1\right) d s+\int_{t_{1}}^{t}\left(R_{\sigma}(s)-1\right) d s\right]$. Generally, for $t \in\left(t_{k-1}, t_{k}\right]$, it can be shown that

$$
\begin{aligned}
& E\left[I_{1}(t)+I_{2}(t)\right] \\
& \quad \leq \frac{1}{\theta}\left(I_{1}\left(t_{0}\right)+I_{2}\left(t_{0}\right)\right) \exp \left\{\left[\int_{t_{0}}^{t_{1}}\left(R_{\sigma}(s)-1\right) d s+\cdots+\int_{t_{k-1}}^{t}\left(R_{\sigma}(s)-1\right) d s\right]\right\} .
\end{aligned}
$$

Since $\bar{R}_{0}<1$, it can be obtained that $\zeta=\exp \left\{\left[\int_{t_{0}}^{t_{1}}\left(R_{\sigma}(s)-1\right) d s+\cdots+\int_{t_{k-1}}^{t}\left(R_{\sigma}(s)-1\right) d s\right]\right\}<1$. Note that $I_{1}, I_{2} \geq 0$, and hence $I_{1}$ and $I_{2}$ converge to zero exponentially. Furthermore, we can get that $A$ converges to zero. As a consequence, $Q_{0}=(\lambda / a, 0,0,0,0)$ is stochastically asymptotically stable, which implies that the disease in system (1) will go to extinction with probability 1 .

Remark 1 From Theorem 1, it can be shown that if $I_{1}$ and $I_{2}$ approach zero exponentially with probability 1 , then $A$ converges to zero with probability 1 , and $S$ converges to $\frac{\lambda}{a}$ with probability 1 , which implies that $Q_{0}=(\lambda / a, 0,0,0)$ is stochastically asymptotically stable even if some subsystems are unstable.

Remark 2 In the special case that two types of noise and distributed time delay are absent and all coefficients are constants, system (1) degenerates into the following:

$$
\left\{\begin{array}{l}
d S(t)=\left\{\lambda-p S(t) I_{1}(t)-q S(t) I_{2}(t)-a S(t)\right\} d t, \\
d I_{1}(t)=\left\{p S(t) I_{1}(t)+q S(t) I_{2}(t)-\left(r+a+\rho^{1}\right) I_{1}(t)\right\} d t, \\
d I_{2}(t)=\left\{e r I_{1}(t)-\left(a+b+\rho^{2}\right) I_{2}(t)\right\} d t, \\
d A(t)=\left\{(1-e) r I_{1}(t)+\rho^{2} I_{2}(t)-c A(t)-a A(t)\right\} d t .
\end{array}\right.
$$

Obviously, the basic reproduction number $\bar{R}_{0}$ of system (1) is reduced to $R_{0}$ given in some references (see [14, 24, 35]). From Theorem 1, it is easily found that the main results in these references are improved and extended in this paper.

Next, we study the eradication of the disease with probability 1 when a switching signal for system (1) is periodic. Assume that the periodic switching signal is constructed the same as that of ref. [41]: (1) $t_{k}-t_{k-1}=\omega_{k}$ with $\omega_{k+m}=\omega_{k}$, in which $\omega=\omega_{1}+\cdots+\omega_{m}$ is one period of the switching signal; (2) $z_{\sigma}\left(z=p, q, r, \rho^{1}, \rho^{2}, e, b, c\right)$ is a switching parameter which satisfies $z_{i}=z_{k}$ for $t \in\left(t_{k-1}, t_{k}\right], z_{k+m}=z_{k}$; and $z_{k}(t)=z_{k}(t+\omega)$. $\mathcal{I}_{\text {periodic }}$ is the set of periodic switching rules and $\mathcal{I}_{\text {periodic }} \subset \mathcal{I}$. Hence, we obtain the following theorem.

Theorem 2 Suppose that the switching signal $\sigma$ is periodic, and $a>\Lambda$. Suppose that

$$
\hat{R}_{0}=\frac{\int_{t_{0}}^{t_{0}+\omega}\left[\lambda \tau q_{\sigma} B_{\sigma}+e_{\sigma} r_{\sigma}(a-\Lambda) D_{\sigma}\right] d s}{(a-\Lambda) \omega},
$$


with $B_{\sigma}=\frac{a-\Lambda}{\lambda \tau\left(q_{\sigma}-p_{\sigma}\right)+(a-\Lambda)\left(r_{\sigma}+a+\rho_{\sigma}^{1}\right)}>0$, and $D_{\sigma}=\frac{1}{e_{\sigma} r_{\sigma}+a-b_{\sigma}-\rho_{\sigma}^{2}}>0$. If $\hat{R}_{0}<1$, then $Q_{0}=$ $(\lambda / a, 0,0,0,0)$ is stochastically asymptotically stable, which implies that the disease will go to extinction with probability 1.

Proof By the proof preceding Theorem 1, it suffices to show that, for $t=t_{0}+\omega$,

$$
\begin{aligned}
E & {\left[I_{1}\left(t_{0}+\omega\right)+I_{2}\left(t_{0}+\omega\right)\right] } \\
& \leq \frac{1}{\theta} E\left[I_{1}\left(t_{0}\right)+I_{2}\left(t_{0}\right)\right] \exp \left\{\left[\int_{t_{0}}^{t_{1}}\left(R_{1}-1\right) d s+\cdots+\int_{t_{m-1}}^{t_{m}}\left(R_{m}-1\right) d s\right]\right\} \\
& \leq \frac{1}{\theta} I_{1}\left(t_{0}\right)+I_{2}\left(t_{0}\right) \zeta
\end{aligned}
$$

in which $\zeta=\exp \left\{\left[\int_{t_{0}}^{t_{1}}\left(R_{1}-1\right) d s+\cdots+\int_{t_{m-1}}^{t_{m}}\left(R_{m}-1\right) d s\right]\right\}$. Taking integer $h=1,2, \ldots$, it follows that $E\left[I_{1}\left(t_{0}+h \omega\right)+I_{2}\left(t_{0}+h \omega\right)\right] \leq \frac{1}{\theta} \zeta E\left[I_{1}\left(t_{0}+(h-1) \omega\right)+I_{2}\left(t_{0}+(h-1) \omega\right)\right] \leq \cdots \leq$ $\frac{1}{\theta} \zeta^{h} E\left[I_{1}\left(t_{0}\right)+I_{2}\left(t_{0}\right)\right]$. Thus, when $h$ approaches infinity, the sequence $\left\{E\left[I_{1}\left(t_{0}+h \omega\right)+I_{2}\left(t_{0}+\right.\right.\right.$ $h \omega)]\}$ converges to zero. In general, for $t \in\left(t_{k-1}, t_{k}\right]$ and $t_{0}+h \omega<t_{k} \leq t_{0}+(h+1) \omega$, we can obtain that $E\left[I_{1}(t)+I_{2}(t)\right] \leq \frac{1}{\theta} E\left[I_{1}\left(t_{0}+h \omega\right)+I_{2}\left(t_{0}+h \omega\right)\right] \exp \left[\int_{t_{0}+h \omega}^{t}\left(R_{\sigma}(s)-1\right) d s\right] \leq$ $\frac{1}{\theta} M E\left[I_{1}\left(t_{0}+h \omega\right)+I_{2}\left(t_{0}+h \omega\right)\right]$, where $M=\max _{t_{0}+h \omega<t \leq t_{0}+(h+1) \omega} \exp \left[\int_{t_{0}+h \omega}^{t}\left(R_{\sigma}(s)-1\right) d s\right]$. Note that $I_{1}, I_{2} \geq 0$, and hence $I_{1}$ and $I_{2}$ approach zero exponentially, which implies that $A$ converges to zero, $S$ converges to $\lambda / a$. Therefore, $Q_{0}=(\lambda / a, 0,0,0,0)$ is stochastically asymptotically stable. In other words, the disease will die out with probability 1 .

Remark 3 Given an amplitude of bounded noise $\Lambda$, the condition $\hat{R}_{0}<1$ in Eq. (19) defines a time delay $\tau_{c}$ such that $\tau>\tau_{c}$ guarantees the disappearance of the disease. Similarly, given a time delay $\tau$, the condition $\hat{R}_{0}<1$ in Eq. (19) defines an amplitude of bounded noise $\Lambda_{c}$.

Next, we present some threshold conditions to show that the disease is weakly persistent in system (1).

\section{Theorem 3 Assume that}

$$
\tilde{R}_{0}=\sup _{t \geq l} \int_{t_{0}}^{t}\left(X_{\sigma} q_{\sigma} G \tau+e_{\sigma} r_{\sigma} Y_{\sigma}\right) d s
$$

for some $l \geq t_{0}$, in which $G$ is given in Proposition 3, $X_{\sigma}=\frac{1}{G \tau\left(q_{\sigma}-p_{\sigma}\right)+\left(a+r_{\sigma}+\rho_{\sigma}^{1}\right)}>0$, and $Y_{\sigma}=$ $\frac{1}{e_{\sigma} r_{\sigma}+a-b_{\sigma}+\rho_{\sigma}^{2}}>0$. If $\tilde{R}_{0}>1$, then the disease is weakly persistent with probability 1.

Proof Let us consider the following set of Lyapunov functions:

$$
\begin{aligned}
V_{\sigma}= & X_{\sigma} I_{1}(t)+Y_{\sigma} I_{2}(t)+X_{\sigma} p_{\sigma} \int_{0}^{\tau} \int_{t-h}^{t} S(u+h) I_{1}(u) d u d h \\
& +X_{\sigma} q_{\sigma} \int_{0}^{\tau} \int_{t-h}^{t} S(u+h) I_{2}(u) d u d h,
\end{aligned}
$$

where $X_{\sigma}$ and $Y_{\sigma}$ are defined in the theorem. 
Then we can compute the time derivative of $V_{\sigma}(t)$ along system $(1)$ as follows:

$$
\begin{aligned}
d V_{\sigma}= & X_{\sigma} d I_{1}(t)+Y_{\sigma} d I_{2}(t)+X_{\sigma} p_{\sigma} \int_{0}^{\tau}\left\{S(t+h) I_{1}(t)-S(t) I_{1}(t-h)\right\} d h d t \\
& +X_{\sigma} q_{\sigma} \int_{0}^{\tau}\left\{S(t+h) I_{2}(t)-S(t) I_{2}(t-h)\right\} d h d t \\
= & X_{\sigma}\left\{-\left(r_{\sigma}+a+\rho_{\sigma}^{1}\right) I_{1}(t)+p_{\sigma} \int_{0}^{\tau} S(t+h) I_{1}(t) d h\right. \\
& \left.+q_{\sigma} \int_{0}^{\tau} S(t+h) I_{2}(t) d h\right\} d t+Y_{\sigma}\left\{e_{\sigma} r_{\sigma} I_{1}(t)\right. \\
& \left.-\left(a+b_{\sigma}+\rho_{\sigma}^{2}\right) I_{2}(t)\right\} d t-X_{\sigma} \varphi_{1} I_{1}(t) d W_{1}(t)-Y_{\sigma} \varphi_{2} I_{2}(t) d W_{2}(t) \\
\geq & \left\{X_{\sigma}\left(p_{\sigma} G \tau-r_{\sigma}-a-\rho_{\sigma}^{1}\right) I_{1}(t)+Y_{\sigma} e_{\sigma} r_{\sigma}\right\} I_{1}(t) d t+\left\{X_{\sigma} q_{\sigma} G \tau\right. \\
& \left.-Y_{\sigma}\left(a+b_{\sigma}+\rho_{\sigma}^{2}\right) I_{2}(t)\right\} d t-X_{\sigma} \varphi_{1} I_{1}(t) d W_{1}(t)-Y_{\sigma} \varphi_{2} I_{2}(t) d W_{2}(t) \\
\geq & \left(\tilde{R}_{\sigma}-1\right)\left(I_{1}(t)+I_{2}(t)\right) d t-X_{\sigma} \varphi_{1} I_{1}(t) d W_{1}(t)-Y_{\sigma} \varphi_{2} I_{2}(t) d W_{2}(t),
\end{aligned}
$$

where $\tilde{R}_{\sigma}=X_{\sigma} q_{\sigma} G \tau+e_{\sigma} r_{\sigma} Y_{\sigma}$. Then, integrating Eq. (23) over $\left[t_{k-1}, t_{k}\right]$, it can be calculated that

$$
\begin{aligned}
V(t) \geq & V\left(t_{k-1}\right)+\int_{t_{k-1}}^{t}\left\{\left(\tilde{R}_{\sigma}(s)-1\right) I_{1}(s) d s-X_{\sigma}(s) \varphi_{1} I_{1}(s) d W_{1}(s)\right\} \\
& +\int_{t_{k-1}}^{t}\left\{\left(\tilde{R}_{\sigma}(s)-1\right) I_{2}(s) d s-Y_{\sigma} \varphi_{2} I_{2}(s) d W_{2}(t)\right\} .
\end{aligned}
$$

Taking the expectation for Eq. (24), it follows that

$$
\begin{aligned}
E[V(t)] \geq & E\left[V\left(t_{k-1}\right)\right]+E\left[\int_{t_{k-1}}^{t}\left\{\left(\tilde{R}_{\sigma}(s)-1\right) I_{1}(s) d s-X_{\sigma}(s) \varphi_{1} I_{1}(s) d W_{1}(s)\right\}\right] \\
& +E\left[\int_{t_{k-1}}^{t}\left\{\left(\tilde{R}_{\sigma}(s)-1\right) I_{2}(s) d s-Y_{\sigma} \varphi_{2} I_{2}(s) d W_{2}(t)\right\}\right] \\
\geq & E\left[V\left(t_{k-1}\right)\right]+\int_{t_{k-1}}^{t}\left(\tilde{R}_{\sigma}(s)-1\right) E\left[I_{1}(s)+I_{2}(s)\right] d s .
\end{aligned}
$$

Hence, we obtain the following results:

$$
E\left[\frac{d V(t)}{d t}\right] \geq\left(\tilde{R}_{\sigma}-1\right) E\left[I_{1}(t)+I_{2}(t)\right]
$$

Letting $\delta=\max _{\sigma \in\{1,2, \ldots, m\}}\left\{X_{\sigma}\left(\frac{\lambda \tau}{a-\Lambda} p_{\sigma}-r_{\sigma}-a-\rho_{\sigma}^{1}\right)+Y_{\sigma} e_{\sigma} r_{\sigma}, X_{\sigma} q_{\sigma} \frac{\lambda \tau}{a-\Lambda}-Y_{\sigma}\left(a+b_{\sigma}+\rho_{\sigma}^{2}\right)\right\}$, it follows that

$$
\left(\tilde{R}_{\sigma}-1\right) E\left[I_{1}(t)+I_{2}(t)\right] \leq E\left[\frac{d V(t)}{d t}\right] \leq \delta \frac{d}{d t} E\left[I_{1}(t)+I_{2}(t)\right]
$$

i.e.,

$$
\delta \frac{d}{d t} E\left[I_{1}(t)+I_{2}(t)\right] \geq\left(R_{\sigma}-1\right) E\left[I_{1}(t)+I_{2}(t)\right]
$$


Moreover, we have that $E\left[I_{1}(t)+I_{2}(t)\right] \geq E\left[I_{1}\left(t_{k-1}\right)+I_{2}\left(t_{k-1}\right)\right] \exp \left[\int_{t_{k-1}}^{t} \frac{1}{\delta}\left(\tilde{R}_{\sigma}(s)-1\right) d s\right]$. Then we obtain that, for $t \in\left(0, t_{1}\right], E\left[I_{1}(t)+I_{2}(t)\right] \geq\left(I_{1}\left(t_{0}\right)+I_{2}\left(t_{0}\right)\right) \exp \left[\int_{t_{0}}^{t} \frac{1}{\delta}\left(\tilde{R}_{\sigma}(s)-1\right) d s\right]$. For $t \in\left(t_{1}, t_{2}\right]$, it can be derived that $E\left[I_{1}(t)+I_{2}(t)\right] \geq\left(I_{1}\left(t_{0}\right)+I_{2}\left(t_{0}\right)\right) \exp \left[\int_{t_{0}}^{t_{1}} \frac{1}{\delta}\left(\tilde{R}_{\sigma}(s)-1\right) d s+\right.$ $\left.\int_{t_{1}}^{t} \frac{1}{\delta}\left(\tilde{R}_{\sigma}(s)-1\right) d s\right]$. In general, for $t \in\left(t_{k-1}, t_{k}\right]$, it can be shown that

$$
\begin{aligned}
& E\left[I_{1}(t)+I_{2}(t)\right] \\
& \quad \geq\left(I_{1}\left(t_{0}\right)+I_{2}\left(t_{0}\right)\right) \exp \left\{\frac{1}{\delta}\left[\int_{t_{0}}^{t_{1}}\left(\tilde{R}_{\sigma}(s)-1\right) d s+\cdots+\int_{t_{k-1}}^{t}\left(\tilde{R}_{\sigma}(s)-1\right) d s\right]\right\} .
\end{aligned}
$$

On the other hand, $\tilde{R}_{0}>1$ implies that $\varsigma=\exp \left\{\frac{1}{\theta}\left[\int_{t_{0}}^{t_{1}}\left(R_{\sigma}(s)-1\right) d s+\cdots+\int_{t_{k-1}}^{t}\left(R_{\sigma}(s)-\right.\right.\right.$ 1) $d s]\}>1$ such that $\lim _{t \rightarrow \infty} E\left(I_{1}(t)+I_{2}(t)\right) \rightarrow \infty$ as $t \rightarrow \infty$. Therefore, the disease weakly persists with probability 1 .

Remark 4 It is worthy to point out from Theorem 3 that the disease is weakly persistent with $\tilde{R}_{0}>1$.

Theorem 4 Suppose that the switching signal $\sigma$ is periodic. Suppose that

$$
\tilde{R}_{0}=\int_{t_{0}}^{t_{0}+\omega}\left(X_{\sigma} q_{\sigma} G \tau+e_{\sigma} r_{\sigma} Y_{\sigma}\right) d s
$$

with G given in Proposition 3, $X_{\sigma}=\frac{1}{G \tau\left(q_{\sigma}-p_{\sigma}\right)+\left(a+r_{\sigma}+\rho_{\sigma}^{1}\right)}>0$, and $Y_{\sigma}=\frac{1}{e_{\sigma} r_{\sigma}+a-b_{\sigma}+\rho_{\sigma}^{2}}>0$. If $R_{0}^{*}>1$, then the disease is weakly persistent with probability 1 .

Proof From the proof preceding to Theorem 3, we have that, for $t=t_{0}+\omega$,

$$
\begin{aligned}
& E\left[I_{1}\left(t_{0}+\omega\right)+I_{2}\left(t_{0}+\omega\right)\right] \\
& \quad \geq E\left[I_{1}\left(t_{0}\right)+I_{2}\left(t_{0}\right)\right] \exp \left\{\frac{1}{\delta}\left[\int_{t_{0}}^{t_{1}}\left(\tilde{R}_{1}-1\right) d s+\cdots+\int_{t_{m-1}}^{t_{m}}\left(\tilde{R}_{m}-1\right) d s\right]\right\} \\
& \quad \geq I_{1}\left(t_{0}\right)+I_{2}\left(t_{0}\right) \varsigma .
\end{aligned}
$$

For some integer $h=1,2, \ldots, E\left[I_{1}\left(t_{0}+h \omega\right)+I_{2}\left(t_{0}+h \omega\right)\right] \geq \varsigma E\left[I_{1}\left(t_{0}+(h-1) \omega\right)+I_{2}\left(t_{0}+(h-\right.\right.$ 1) $\omega)] \geq \cdots \geq \varsigma^{h} E\left[I_{1}\left(t_{0}\right)+I_{2}\left(t_{0}\right)\right]$, which implies that when $h$ approaches $\infty$, the sequence $\left\{E\left[I_{1}\left(t_{0}+h \omega\right)+I_{2}\left(t_{0}+h \omega\right)\right]\right\}$ converges to $\infty$. Thus, for $t \in\left(t_{k-1}, t_{k}\right]$ and $t_{0}+h \omega<t_{k} \leq$ $t_{0}+(h+1) \omega$, we derive that $E\left[I_{1}(t)+I_{2}(t)\right] \geq E\left[I_{1}\left(t_{0}+h \omega\right)+I_{2}\left(t_{0}+h \omega\right)\right] \exp \left[\int_{t_{0}+h \omega}^{t} \frac{1}{\delta}\left(\tilde{R}_{\sigma}(s)-\right.\right.$ 1) $d s] \geq M E\left[I_{1}\left(t_{0}+h \omega\right)+I_{2}\left(t_{0}+h \omega\right)\right]$, in which $M=\min _{t_{0}+h \omega \leq t \leq t_{0}+(h+1) \omega} \exp \left[\int_{t_{0}+h \omega}^{t} \frac{1}{\delta}\left(\tilde{R}_{\sigma}(s)-\right.\right.$ 1) $d s$ ]. It can be derived that $\lim _{t \rightarrow \infty} E\left(I_{1}(t)+I_{2}(t)\right) \rightarrow \infty$. Therefore, the disease is weakly persistent with probability 1 .

Remark 5 It has been shown in Theorem 4 that the basic reproduction number $\tilde{R}_{0}$ is related to the transmission mechanism of disease, and the disease is weakly persistent even if the basic reproduction number of some subsystem is less than one.

Remark 6 We observe from the results of Theorem 2 and Theorem 4 that two types of noise and time delay have an important effect on the dynamics of the proposed model. 


\section{Pulse control schemes}

In this section, we develop pulse control strategies to eliminate the disease. New threshold conditions are established to guarantee that pulse control strategies are successful in clearing up the disease.

\subsection{Pulse treatment of two types of the infected individuals}

In this subsection, we discuss the case that the pulse treatment strategy is applied to the asymptomatic infected individuals and the symptomatic infected individuals in a relatively short time period compared with the dynamics of the disease. Immediately after each pulse treatment, the disease evolves from its new initial state without being further affected by the treatment scheme until the next pulse is applied [17,31]. At each treatment time, assume that the asymptomatic infected individuals and the symptomatic infected individuals are provided with pulse treatment at the same time, and $v(0<v<1)$ is a fraction of the asymptomatic infected individuals and the symptomatic infected individuals who are in pulse therapy, respectively. And assume that two types of the treated population can become died out. Suppose that we ignore the effect caused by drug to other individuals. When pulse treatment strategies are incorporated into system (1), the system may be rewritten as follows:

$$
\left\{\begin{aligned}
d S(t)= & \left\{\lambda-p_{\sigma} S(t) \int_{0}^{\tau} I_{1}(t-h) d h-q_{\sigma} S(t) \int_{0}^{\tau} I_{2}(t-h) d h-a S(t)\right\} d t \\
& -S(t) d M(t), \quad t \neq t_{0}+n \omega, \\
d I_{1}(t)= & \left\{p_{\sigma} S(t) \int_{0}^{\tau} I_{1}(t-h) d h+q_{\sigma} S(t) \int_{0}^{\tau} I_{2}(t-h) d h-\left(r_{\sigma}+a+\rho_{\sigma}^{1}\right) I_{1}(t)\right\} d t \\
& -\varphi_{1} I_{1}(t) d W_{1}(t), \\
d I_{2}(t)= & \left\{e_{\sigma} r_{\sigma} I_{1}(t)-\left(a+b_{\sigma}+\rho_{\sigma}^{2}\right) I_{2}(t)\right\} d t-\varphi_{2} I_{2}(t) d W_{2}(t), \\
d A(t)= & \left\{\left(1-e_{\sigma}\right) r_{\sigma} I_{1}(t)+\rho_{\sigma}^{2} I_{2}(t)-c_{\sigma} A(t)-a A(t)\right\} d t-\varphi_{3} A(t) d W_{3}(t), \\
S\left(t^{+}\right)= & S(t), \quad t=t_{0}+n \omega, \\
I_{1}\left(t^{+}\right)= & (1-v) I_{1}(t), \\
I_{2}\left(t^{+}\right)= & (1-v) I_{2}(t), \\
A\left(t^{+}\right)= & A(t),
\end{aligned}\right.
$$

in which all parameters $\lambda, p_{\sigma}, q_{\sigma}, a, r_{\sigma}, \rho_{\sigma}^{1}, e_{\sigma}, b_{\sigma}, \rho_{\sigma}^{2}$, and $c_{\sigma}$ are defined the same as those of system (1). The switching rule $\sigma \in \mathcal{I}_{\text {periodic-pulse, }}$, where $\mathcal{I}_{\text {periodic-pulse }}$ is the set of periodic switching signals of the above model, and one period is denoted by $\omega=\omega_{1}+\cdots+\omega_{m}$ and $\omega_{k}=t_{k}-t_{k-1}$. Suppose that $S\left(t_{0}^{+}\right)=S\left(t_{0}\right), I_{1}\left(t_{0}^{+}\right)=I_{1}\left(t_{0}\right), I_{2}\left(t_{0}^{+}\right)=I_{2}\left(t_{0}\right)$, and $A\left(t_{0}^{+}\right)=A\left(t_{0}\right)$ are the initial condition of system (32). When there are no noise terms and pulse treatment terms, system (32) has a disease-free equilibrium $Q_{0}=(\lambda / a, 0,0,0,0)$. In the following, we investigate that the disease in system (32) will die out with probability 1 . That is, if $I_{1}=0$ and $I_{2}=0$, then it is obtained that $A$ approaches zero, which means that the disease could be eradicated theoretically.

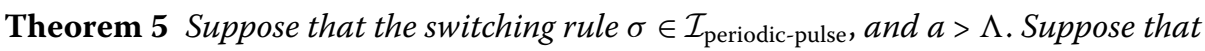

$$
\check{R}_{0}=\frac{\ln (1-v)}{\omega}+\frac{\int_{t_{0}}^{t_{0}+\omega}\left[\lambda \tau q_{\sigma} B_{\sigma}+e_{\sigma} r_{\sigma}(a-\Lambda) D_{\sigma}\right] d s}{(a-\Lambda) \omega}
$$


with $B_{\sigma}=\frac{a-\Lambda}{\lambda \tau\left(q_{\sigma}-p_{\sigma}\right)+(a-\Lambda)\left(r_{\sigma}+a+\rho_{\sigma}^{1}\right)}$, and $D_{\sigma}=\frac{1}{\epsilon_{\sigma} r_{\sigma}+a-b_{\sigma}-\rho_{\sigma}^{2}}$. If $\check{R}_{0}<1$, then $Q_{0}=(\lambda / a, 0,0,0,0)$ is stochastically asymptotically stable. In other words, the disease will go to extinction with probability 1.

Proof From the proof of Theorem 1, we have that, for $t \in\left(t_{k-1}, t_{k}\right]$,

$$
E\left[I_{1}(t)+I_{2}(t)\right] \leq \frac{1}{\theta} E\left[I_{1}\left(t_{k-1}\right)+I_{2}\left(t_{k-1}\right)\right] \exp \left[\int_{t_{k-1}}^{t}\left(R_{\sigma}(s)-1\right) d s\right]
$$

where $\theta=\min _{\sigma \in\{1,2, \ldots, m\}}\left\{B_{\sigma}, D_{\sigma}\right\}$ and $R_{\sigma}=\frac{\lambda \tau q_{\sigma} B_{\sigma}+e_{\sigma} r_{\sigma}(a-\Lambda) D_{\sigma}}{a-\Lambda}$. Then it can be shown that, for $t \in\left(0, t_{1}\right], E\left[I_{1}(t)+I_{2}(t)\right] \leq \frac{1}{\theta}\left(I_{1}\left(t_{0}\right)+I_{2}\left(t_{0}\right)\right) \exp \left[\int_{t_{0}}^{t}\left(R_{\sigma}(s)-1\right) d s\right]$. Moreover, we can observe that, for $t \in\left(t_{k-1}, t_{k}\right], E\left[I_{1}(t)+I_{2}(t)\right] \leq \frac{1}{\theta}\left(I_{1}\left(t_{0}\right)+I_{2}\left(t_{0}\right)\right) \exp \left\{\int_{t_{0}}^{t_{1}}\left(R_{\sigma}(s)-1\right) d s+\int_{t_{1}}^{t_{2}}\left(R_{\sigma}(s)-\right.\right.$ 1) $\left.d s+\cdots+\int_{t_{k-1}}^{t}\left(R_{\sigma}(s)-1\right) d s\right\}$.

Immediately after the pulse switch time $t=t_{0}+\omega$, it follows that

$$
\begin{aligned}
E[ & \left.I_{1}\left(t_{0}+\omega\right)^{+}+I_{2}\left(t_{0}+\omega\right)^{+}\right] \\
& =(1-v) E\left[I_{1}\left(t_{0}+\omega\right)+I_{2}\left(t_{0}+\omega\right)\right] \\
& \leq \frac{1}{\theta}(1-v)\left(I_{1}\left(t_{0}\right)+I_{2}\left(t_{0}\right)\right) \exp \left\{\int_{t_{0}}^{t_{1}}\left(R_{\sigma}(s)-1\right) d s+\cdots+\int_{t_{m-1}}^{t_{m}}\left(R_{\sigma}(s)-1\right) d s\right\} \\
& \leq \frac{1}{\theta}\left(I_{1}\left(t_{0}\right)+I_{2}\left(t_{0}\right)\right) \exp \left\{\ln (1-v)+\int_{t_{0}}^{t_{0}+\omega}\left(R_{\sigma}(s)-1\right) d s\right\} .
\end{aligned}
$$

$\check{R}_{0}<1$ implies that $\tilde{\zeta}=\exp \left\{\ln (1-v)+\int_{t_{0}}^{t_{0}+\omega}\left(R_{\sigma}(s)-1\right) d s\right\}<1$. Since the switching signal $\sigma$ is periodic, we can derive that $E\left[I_{1}\left(t_{0}+h \omega\right)^{+}+I_{2}\left(t_{0}+h \omega\right)^{+}\right] \leq \cdots \leq \frac{1}{\theta}\left(I_{1}\left(t_{0}\right)+I_{2}\left(t_{0}\right)\right) \tilde{\zeta}^{h}$ for some integer $h=1,2, \ldots$. Thus, it can obtained that when $h$ approaches $\infty$, the sequence $\left\{E\left[I_{1}\left(t_{0}+h \omega\right)^{+}+I_{2}\left(t_{0}+h \omega\right)^{+}\right]\right\}$approaches 0 . In general, for $t \in\left(t_{k-1}, t_{k}\right]$ and $t_{0}+h \omega<t_{k} \leq$ $t_{0}+(h+1) \omega$, it follows that $E\left[I_{1}(t)+I_{2}(t)\right] \leq \frac{1}{\theta} E\left[I_{1}\left(t_{0}+h \omega\right)^{+}+I_{2}\left(t_{0}+h \omega\right)^{+}\right] \exp \left[\int_{t_{0}+h \omega}^{t}\left(R_{\sigma}(s)-\right.\right.$ 1) $d s] \leq \frac{1}{\theta} M E\left[I_{1}\left(t_{0}+h \omega\right)^{+}+I_{2}\left(t_{0}+h \omega\right)^{+}\right]$, where $M=\max _{t_{0}+h \omega<t \leq t_{0}+(h+1) \omega} \exp \left[\int_{t_{0}+h \omega}^{t}\left(R_{\sigma}(s)-\right.\right.$ 1) $d s$ ]. It can be shown that $I_{1}$ and $I_{2}$ approach zero with probability 1 . Furthermore, we can get that $A$ approaches zero with probability 1 . Therefore, $Q_{0}=(\lambda / a, 0,0,0,0)$ is stochastically asymptotically stable, i.e., the disease will go to extinction with probability 1.

Remark 7 Theorem 5 shows that the disease can be eradicated when the basic reproduction number related to two types of noise, time delay, and pulse treatment is less than one. Given an amplitude of bounded noise $\Lambda$, the condition $\breve{R}_{0}<1$ in Theorem 5 defines a vaccination $\tau_{c}$ such that $v>v_{c}$ guarantees eradication of the disease, where

$$
v_{c}=1-\exp \left\{\omega-\frac{\int_{t_{0}}^{t_{0}+\omega}\left[\lambda \tau q_{\sigma} B_{\sigma}+e_{\sigma} r_{\sigma}(a-\Lambda) D_{\sigma}\right] d s}{a-\Lambda}\right\} .
$$

\subsection{Pulse vaccination of the susceptible individuals and the infected individuals}

The subsection deals with pulse vaccination applied to the susceptible individuals and the infected individuals at the same time. Suppose that the susceptible individuals are provided with pulse vaccination at each vaccination time, and a fraction $\hat{\theta}(0<\hat{\theta}<1)$ of the susceptible individuals are offered a pulse scheme. And suppose that the vaccinated sus- 
ceptible individuals have permanent immunity. Applied to system (32), the pulse vaccination system is modeled as follows:

$$
\left\{\begin{aligned}
d S(t)= & \left\{\lambda-p_{\sigma} S(t) \int_{t-\tau}^{t} I_{1}(h) d h-q_{\sigma} S(t) \int_{t-\tau}^{t} I_{2}(h) d h-a S(t)\right\} d t \\
& -S(t) d M(t), \quad t \neq t_{0}+n \omega, \\
d I_{1}(t)= & \left\{p_{\sigma} S(t) \int_{t-\tau}^{t} I_{1}(h) d h+q_{\sigma} S(t) \int_{t-\tau}^{t} I_{2}(h) d h-\left(r_{\sigma}+a+\rho_{\sigma}^{1}\right) I_{1}(t)\right\} d t \\
& -\varphi_{1} I_{1}(t) d W_{1}(t), \\
d I_{2}(t)= & \left\{e_{\sigma} r_{\sigma} I_{1}(t)-\left(a+b_{\sigma}+\rho_{\sigma}^{2}\right) I_{2}(t)\right\} d t-\varphi_{2} I_{2}(t) d W_{2}(t), \\
d A(t)= & \left\{\left(1-e_{\sigma}\right) r_{\sigma} I_{1}(t)+\rho_{\sigma}^{2} I_{2}(t)-c_{\sigma} A(t)-a A(t)\right\} d t-\varphi_{3} A(t) d W_{3}(t), \\
S\left(t^{+}\right)= & (1-\hat{\theta}) S(t), \quad t=t_{0}+n \omega, \\
I_{1}\left(t^{+}\right)= & (1-v) I_{1}(t), \\
I_{2}\left(t^{+}\right)= & (1-v) I_{2}(t), \\
A\left(t^{+}\right)= & A(t),
\end{aligned}\right.
$$

where all parameters are defined the same as those of system (32) and are nonnegative. The switching signal $\sigma \in \mathcal{I}_{\text {periodic-pulse }}$, where $\mathcal{I}_{\text {periodic-pulse }}$ is the set of period switching signals of (32), and the period is denoted by $\omega=\omega_{1}+\cdots+\omega_{m}$ and $\omega_{k}=t_{k}-t_{k-1}$. Assume that $S\left(t_{0}^{+}\right)=$ $S\left(t_{0}\right)>0, I_{1}\left(t_{0}^{+}\right)=I_{1}\left(t_{0}\right) \geq 0, I_{2}\left(t_{0}^{+}\right)=I_{2}\left(t_{0}\right) \geq 0$, and $A\left(t_{0}^{+}\right)=A\left(t_{0}\right) \geq 0$ are initial conditions of (32). When the average of oscillation for both the asymptomatic infected individuals and the symptomatic infected individuals is zero, it is deduced that $A$ approaches zero. Then system (37) can be simplified to the following limit form:

$$
\left\{\begin{array}{l}
d S(t)=\{\lambda-a S(t)\} d t-S(t) d M(t), \quad t \neq t_{0}+n \omega, \\
S\left(t^{+}\right)=(1-\hat{\theta}) S(t), \quad t=t_{0}+n \omega .
\end{array}\right.
$$

According to Lemma 2.2 of [42], Eq. (38) has a stochastic solution $\widetilde{S}$. So that system (37) has a stochastic solution $\widetilde{Q}=(\widetilde{S}, 0,0,0)$. Next, threshold conditions are derived to show that system (37) is stochastically asymptotically stable, which means that the disease could be cleared out theoretically.

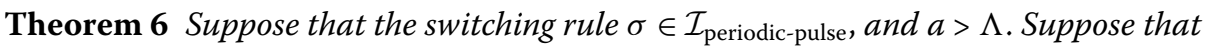

$$
\hat{R}_{0}=\frac{\ln (1-v)}{\omega}+\frac{\int_{t_{0}}^{t_{0}+\omega}\left[\lambda \tau q_{\sigma} B_{\sigma}+e_{\sigma} r_{\sigma}(a-\Lambda) D_{\sigma}\right] d s}{(a-\Lambda) \omega}
$$

with $B_{\sigma}=\frac{a-\Lambda}{\lambda \tau\left(q_{\sigma}-p_{\sigma}\right)+(a-\Lambda)\left(r_{\sigma}+a+\rho_{\sigma}^{1}\right)}$, and $D_{\sigma}=\frac{1}{\epsilon_{\sigma} r_{\sigma}+a-b_{\sigma}-\rho_{\sigma}^{2}}$. If $\hat{R}_{0}<1$, then the solution $\widetilde{Q}=$ $(\widetilde{S}, 0,0,0)$ is stochastically asymptotically stable, which means that the disease will go to extinction with probability 1 .

Proof Suppose that the switching rule $\sigma \in \mathcal{I}_{\text {periodic-pulse. }}$ From the proof of Theorem 5, it follows that $I_{1}(t)$ and $I_{1}(t)$ converge to zero with probability 1 in infinite time, respectively. Moreover, $A(t)$ converges to zero with probability 1 in infinite time. Thus, the limit system with $I_{1}(t)=0, I_{2}(t)=0$ and $A(t)=0$ is given by system (38). Therefore, the solution $\widetilde{Q}=(\widetilde{S}, 0,0,0)$ is stochastically asymptotically stable. In other words, the disease will go to extinction with probability 1 . 


\section{Computer simulations}

This section gives four examples to illustrate the effectiveness of the theoretical results. Suppose that one period is $\omega=1$, the number of subsystems is $m=2$, and $t_{0}=0$, and most of the values are taken from those in $[7,12,14,23,24]$.

Example 1 Consider the dynamics of system (1) with the initial conditions $S(0)=10,000$, $I_{1}(0)=1000, I_{2}(0)=10,000$, and $A(0)=1000$. Assume that the activity time in the 1 st subsystem is $\omega_{1}=0.8$ and the activity time in the 2 nd subsystem is $\omega_{2}=0.2$. Choose constant parameter values $\lambda=10^{3}$ and $a=0.3$. Take the switching parameters $p_{1}=0.01, q_{1}=0.07$, $r_{1}=0.7, \rho_{1}^{1}=0.7, e_{1}=0.02, b_{1}=10^{-4}, \rho_{1}^{2}=0.7, c_{1}=0.02, p_{2}=0.04, q_{2}=0.05, r_{2}=0.025$, $\rho_{1}^{2}=0.015, e_{2}=0.4, b_{2}=10^{-3}, \rho_{2}^{2}=0.5$, and $c_{2}=0.05$. We will investigate the dynamics of the model, and show the effect of distributed time delay and two types of noise on system (1).

(I) Taking the time delay parameter $\tau=0.01$, the amplitude of bounded noise $\Lambda=0.1$, the center frequency of bounded noise $\Omega=0.2$, and the intensity of noises $\varphi=\varphi_{1}=\varphi_{2}=$ $\varphi_{3}=0.5$. It can be calculated that the basic reproduction number of the first subsystem is 0.7915 ; the basic reproduction number of the second subsystem is 1.5870 . From Theorem 2 , we have $\hat{R}_{0}=0.9506<1$, then the disease in system (1) will go to extinction with probability 1 . It is worth noticing that the entire system (1) may be stochastically asymptotically stable although some subsystem is stochastically asymptotically unstable. Figure 1 shows that the disease eventually disappears under the influence of distributed time delay, switching, and two types of noise. Therefore, the simulation results of system (1) guarantee the theoretical results.

(II) In the absence of two types of noise, and taking the time delay parameter $\tau=0.01$, it can be derived that the basic reproduction number is $0.7769(<1)$, and solutions of system (1) without two types of noise are plotted in Fig. 2. It can be shown from Fig. 1 and Fig. 2 that solutions of system (1) fluctuate randomly around the deterministic solutions of the model without two types of noise.

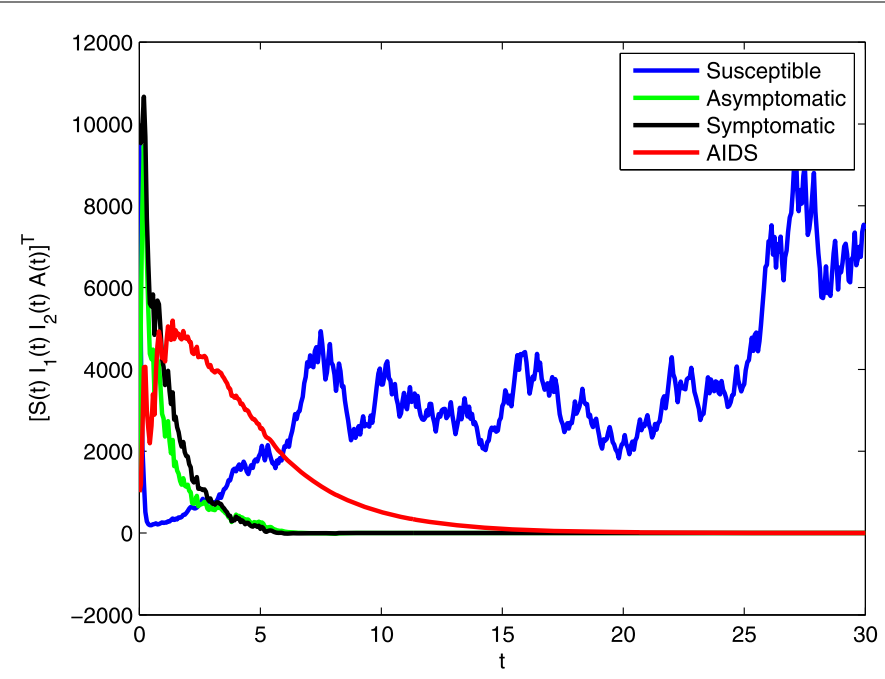

Figure 1 Simulations of system (1) with $\tau=0.01, \Lambda=0.1$, and $\varphi=\varphi_{1}=\varphi_{2}=\varphi_{3}=0.5$ 


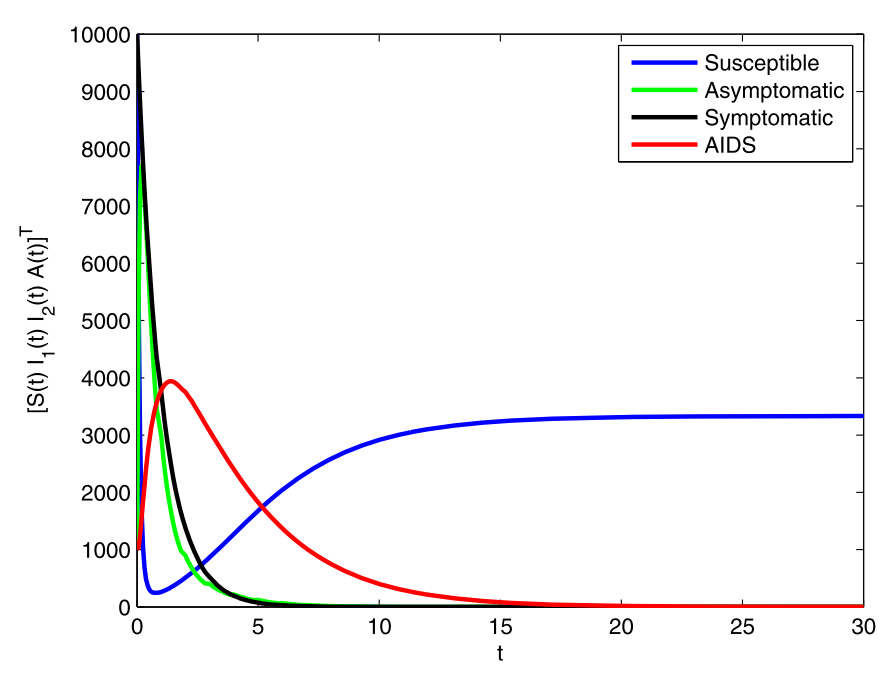

Figure 2 Simulations of system (1) with $\tau=0.01, \Lambda=0$, and $\varphi=\varphi_{1}=\varphi_{2}=\varphi_{3}=0$

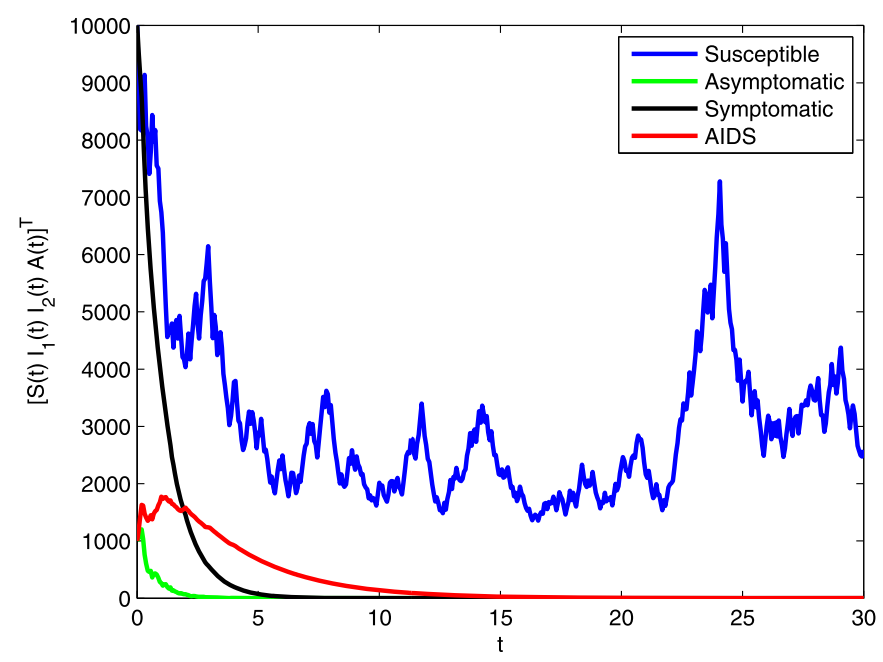

Figure 3 Simulations of system (1) with $\Lambda=0.1, \varphi=\varphi_{1}=\varphi_{2}=\varphi_{3}=0.5$, and $\tau=0$

(III) In the absence of time delay, the amplitude of bounded noise $\Lambda=0.1$, the center frequency of bounded noise $\Omega=0.2$, and the intensity of noises $\varphi=\varphi_{1}=\varphi_{2}=\varphi_{3}=0.5$. It can be derived that the basic reproduction number is $0.0270(<1)$, and numerical simulation results are given in Fig. 3. From both Fig. 1 and Fig. 3, it can be shown that time delay could affect the dynamic behavior of the disease.

Example 2 Consider the persistence of the disease in system (1) with the initial conditions $S(0)=10,000, I_{1}(0)=1000, I_{2}(0)=1000$, and $A(0)=1000$. Assume that the activity time in the 1st subsystem is $\omega_{1}=0.2$ and the activity time in the 2nd subsystem is $\omega_{2}=0.8$, and fix constant values $\lambda=10^{3}$ and $a=0.3$. Take the switching parameters $p_{1}=0.01, q_{1}=0.003$, $r_{1}=0.015, \rho_{1}^{1}=0.0025, e_{1}=0.3, b_{1}=10^{-3}, \rho_{1}^{2}=0.0015, c_{1}=0.002, p_{2}=0.04, q_{2}=0.05$, $r_{2}=0.025, \rho_{1}^{2}=0.0015, e_{2}=0.4, b_{2}=10^{-4}, \rho_{2}^{2}=0.0025$, and $c_{2}=0.005$. Take the time delay 

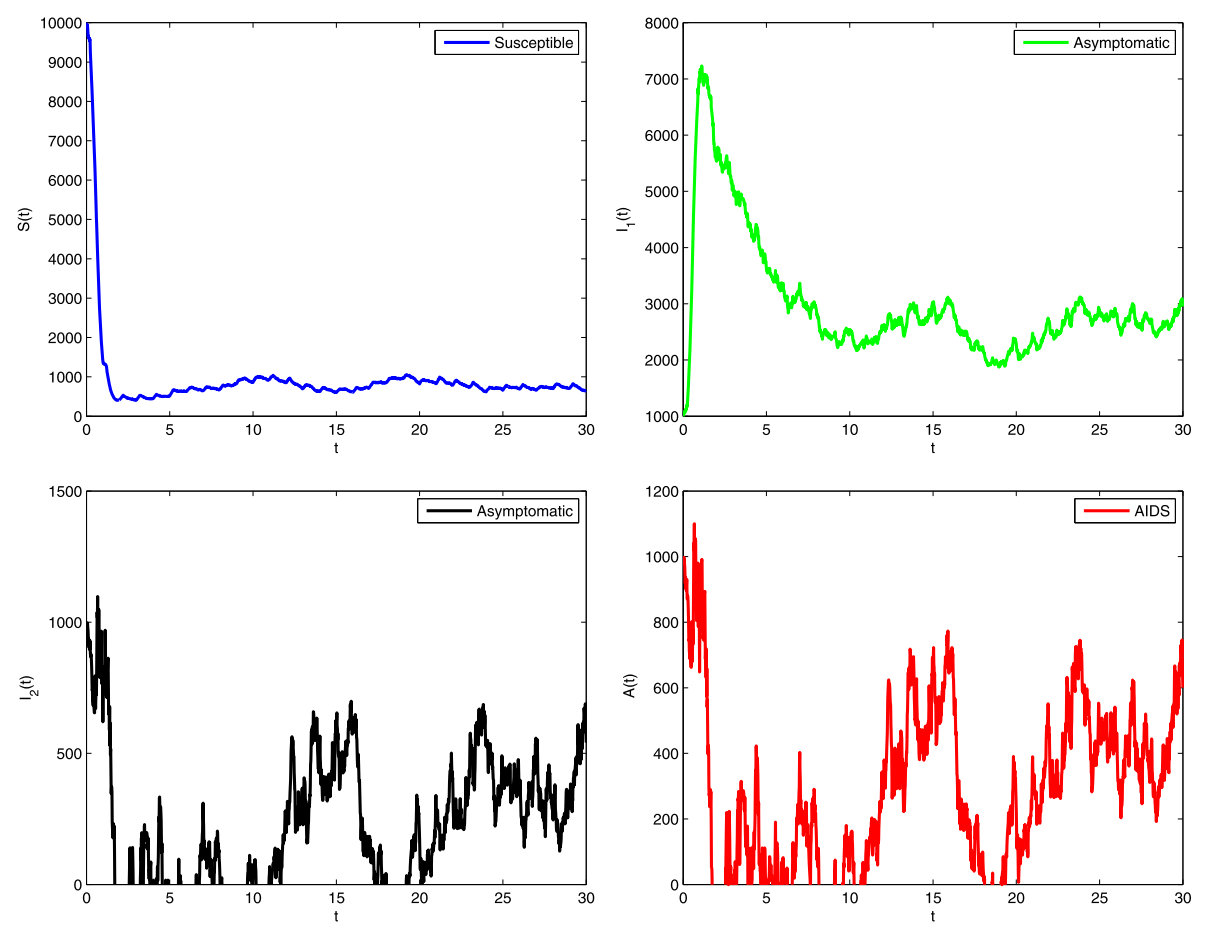

Figure 4 Simulations of system (1) with $\tau=0.01, \Lambda=0.01$, and $\varphi=\varphi_{1}=\varphi_{2}=\varphi_{3}=0.1$

parameter $\tau=0.01, \Lambda=0.01, \Omega=0.2$, and $\varphi=\varphi_{1}=\varphi_{2}=\varphi_{3}=0.1$. It can be computed that the basic reproduction number of the first subsystem is 0.1904 ; the basic reproduction number of the second subsystem is 1.4706 . From Theorem 3 , it can be derived that $\tilde{R}_{0}=$ $1.2146>1$, which implies that the disease persists weakly with probability 1 . It can be seen from Fig. 4 that the disease in system (1) does not appear.

Example 3 Consider the dynamics of system (32) with the initial conditions $S(0)=10,000$, $I_{1}(0)=1000, I_{2}(0)=10,000$, and $A(0)=1000$. Suppose that the other parameters are the same as those of Fig. 4 , and the pulse treatment $v=0.8$. It can be calculated $\check{R}_{0}=0.7460<1$ by Theorem 5 . In comparison with Fig. 4 without pulse treatment, it can be clearly shown from Fig. 5 that the disease can be eradicated with probability 1 due to the effect of the pulse treatment.

Example 4. Consider the dynamics of system (37) with the initial conditions $S(0)=10,000$, $I_{1}(0)=1000, I_{2}(0)=10,000$, and $A(0)=1000$. Assume that the activity time in the 1st subsystem is $\omega_{1}=0.8$ and the activity time in the 2nd subsystem is $\omega_{2}=0.2$, and fix constant values to be $\lambda=10^{3}$ and $a=0.3$. Take the switching parameters $p_{1}=0.01, q_{1}=0.07$, $r_{1}=0.7, \rho_{1}^{1}=0.7, e_{1}=0.02, b_{1}=10^{-4}, \rho_{1}^{2}=0.7, c_{1}=0.02, p_{2}=0.04, q_{2}=0.05, r_{2}=0.025$, $\rho_{1}^{2}=0.015, e_{2}=0.4, b_{2}=10^{-3}, \rho_{2}^{2}=0.5$, and $c_{2}=0.005$. Take the time delay parameters $\tau=0.01, \Lambda=0.1, \Omega=0.2$, and $\varphi=\varphi_{1}=\varphi_{2}=\varphi_{3}=0.5$. Assuming that the pulse vaccination parameters $\theta=0.1$ and $v=0.1$, we can obtain that $\hat{R}_{0}=0.8453<1$ by Theorem 6 . Figure 6 shows that the disease eventually disappears under the conditions of $\hat{R}_{0}<1$. 


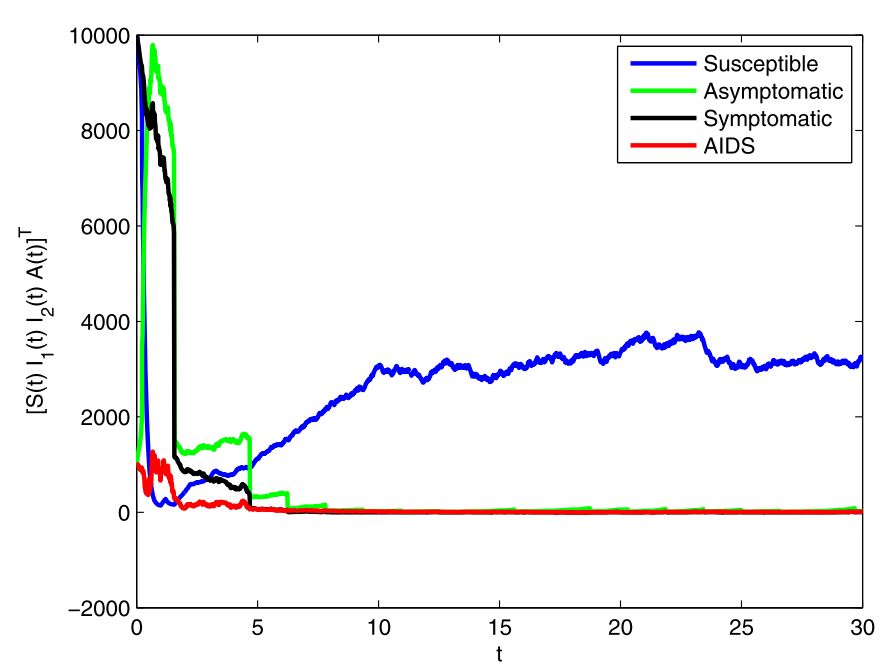

Figure 5 Simulations of system (32) with pulse vaccination parameter $v=0.8$

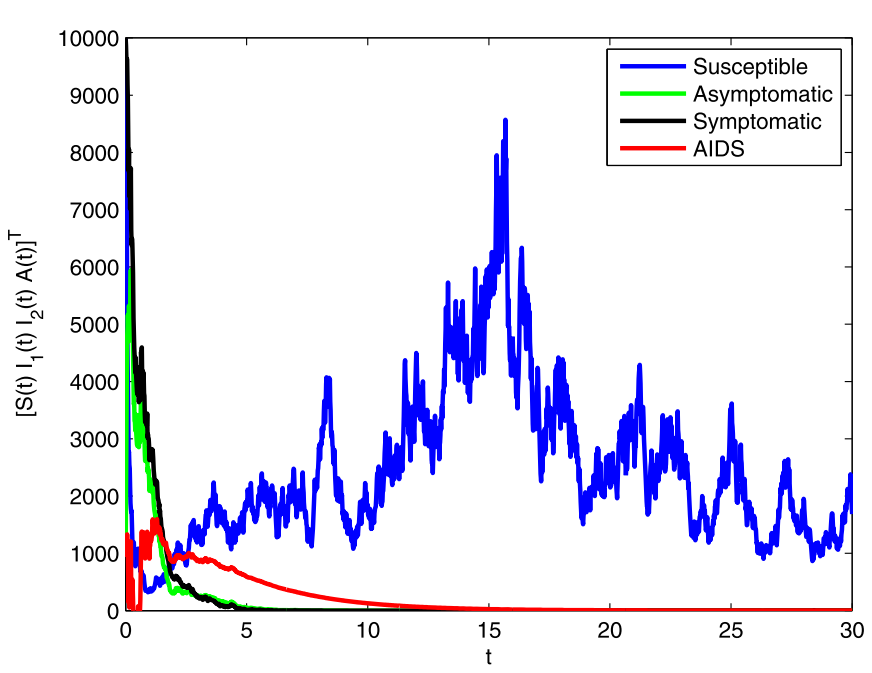

Figure 6 Simulations of system (37) with pulse vaccination parameter $\theta=0.1$ and $v=0.1$

\section{Conclusions}

Pulse vaccination schemes for switched HIV/AIDS epidemic models with distributed time delay and combined bounded noise and Gaussian white noise are developed and investigated in this paper. The model parameters are assumed to be switching parameters, and two types of noise are incorporated into the model on the basis of the method of stochastic perturbation, a switched HIV/AIDS epidemic model with distributed time delay and two types of noise is presented. Novel threshold criteria are developed to check the stochastic asymptotic stability of the disease-free equilibrium of the models via stochastic Itôs lemma and the Lyapunov-Razumikhin method. The results show that the disease is eliminated theoretically under the condition of $\bar{R}_{0}<1$ (given by Theorem 1 ) or $\hat{R}_{0}<1$ (given by Theorem 2 ), which implies that the system is stochastically asymptotically stable regardless of the subsystems being stable or unstable. And the disease could be permanent 
weakly when $\tilde{R}_{0}>1$ (given by Theorem 3 ) or $R_{0}^{*}>1$ (given by Theorem 4 ). Our mathematical analysis suggests that switching parameters, two types of noise, and distributed time delay have significant effect on the spread of the disease. Furthermore, pulse control strategies are applied to two types of the infected population, the susceptible population, and the infected population, respectively. New threshold conditions are derived to examine whether two types of control strategies succeed in the disease elimination or not, i.e., whether $Q_{0}$ or $\widetilde{Q}$ is stochastically asymptotically stable or not. More precisely, a vaccination $\tau_{c}$ is defined to guarantee eradication of the disease if $v>v_{c}$. These results extend the existing work for the corresponding HIV/AIDS model. Simulation examples are provided to verify these results. One future research direction is to study an optimal vaccination strategy for the disease eradication.

\section{Acknowledgements}

The authors are grateful to the referees for their careful reading of this paper and valuable comments.

\section{Funding}

The authors would like to thank the anonymous reviewers and editors for their valuable suggestions and constructive comments, which have helped us to improve the presentation of this work significantly. This work was supported by the National Natural Science Foundation of China (Grant No. 11847081), the Fundamental Research Funds for the Henan Provincial Colleges and Universities in Henan University of Technology (Grant No. 2017QNJH18), and High-Level Personal Foundation of Henan University of Technology (No. 2017BS009).

\section{Availability of data and materials}

Data sharing not applicable to this article as no datasets were generated or analyzed during the current study.

Competing interests

The author declares that they have no competing interests.

Authors' contributions

All authors read and approved the final manuscript.

\section{Publisher's Note}

Springer Nature remains neutral with regard to jurisdictional claims in published maps and institutional affiliations.

Received: 4 November 2020 Accepted: 1 February 2021 Published online: 03 March 2021

\section{References}

1. lannelli, M., Milner, F.A., Pugliese, A., Gonzo, M.: The HIV/AIDS epidemics among drug injectors: a study of contact structure through a mathematical model. Math. Biosci. 139(1), 25-58 (1997)

2. Anderson, R., May, R.: Infectious Diseases of Humans. Dynamics and Control. Oxford University Press, Oxford (1995)

3. Wang, W.H., Ji, C., Bi, Y., Liu, S.: Stability and asymptoticity of stochastic epidemic model with interim immune class and independent perturbations. Appl. Math. Lett. 104, 106245 (2020). https://doi.org/10.1016/j.aml.2020.106245

4. Jia, J., Qin, G.: Stability analysis of HIV/AIDS epidemic model with nonlinear incidence and treatment. Adv. Differ. Equ. (2017). https://doi.org/10.1186/s13662-017-1175-5

5. Asif, M., Jan, S., Haider, N., Al-Mdallal, Q., Abdeljawad, T.: Numerical modeling of NPZ and SIR models with and without diffusion. Results Phys. 19, 103512 (2020). https://doi.org/10.1016/j.rinp.2020.103512

6. Asif, M., Khan, Z., Haider, N., Al-Mdallal, Q.: Numerical simulation for solution of SEIR models by meshless and finite difference methods. Chaos Solitons Fractals 141, 110340 (2020)

7. Anderson, R.M., Medly, G.F., May, R.M., Johnson, A.M.: A preliminary study of the transmission dynamics of the human immunodeficiency virus (HIV), the causative agent of AIDS. IMA J. Math. Appl. Med. Biol. 3, 229-263 (1986)

8. Wang, X.Y., Liu, X.Z., Xu, W., Zhang, K.X.: Stochastic dynamics of HIV models with switching parameters and pulse control. J. Franklin Inst. 352, 2765-2782 (2015)

9. Rihan, F.A., Al-Mdallal, Q.M., AlSakaji, H.J., Hashish, A.A.: Fractional-order epidemic model with time-delay and nonlinear incidence rate. Chaos Solitons Fractals 126, 97-105 (2019)

10. Sharma, S., Samanta, G.P.: Dynamical behaviour of an HIV/AIDS epidemic model. Differ. Equ. Dyn. Syst. 22(4), 369-395 (2014)

11. Saha, S., Samanta, G.P.: Modelling and optimal control of HIV/AIDS prevention through PrEP and limited treatment. Physica A 516, 280-307 (2019)

12. Hsieh, Y.H., Chen, C.H.: Modeling the social dynamics of a sex industry: its implications for spread of HIV/AIDS. Bull. Math. Biol. 66, 143-166 (2004)

13. Naresh, R., Tripathi, A., Omar, S.: Modelling the spread of AIDS epidemic with vertical transmission. Appl. Math. Comput. 178(2), 262-272 (2006)

14. Okosun, K.O., Makinde, O.D., Takaidza, I.: Impact of optimal control on the treatment of HIV/AIDS and screening of unaware infectives. Appl. Math. Model. 37, 3802-3820 (2013) 
15. Earn, D.J., Rohani, P., Bolker, B.M., Grenfell, B.T.: A simple model for complex dynamical transitions in epidemics Science $287,667-670(2000)$

16. Keeling, M.J., Rohani, P., Grenfell, B.T.: Seasonally forced disease dynamics explored as switching between attractors. Physica D 148, 317-335 (2001)

17. Liu, X., Stechlinski, P.: Pulse and constant control schemes for epidemic models with seasonality. Nonlinear Anal., Real World Appl. 12, 931-946 (2011)

18. Liu, J., Liu, X., Xie, W.C.: Input-to-state stability of pulse and switching hybrid systems with time-delay. Automatica 47, 899-908 (2011)

19. Liu, W.: Periodicity of a second-order switched difference system over integers. Appl. Math. Comput. 250, 733-743 (2015)

20. ShenH, S., Park, J.H.: Extended passive filtering for discrete-time singular Markov jump systems with time-varying delays. Signal Process. 128, 68-77 (2016)

21. Li, F., Shen, H.: Finite-time $H_{\infty}$ synchronization control for semi-Markov jump delayed neural networks with randomly occurring uncertainties. Neurocomputing 166, 447-454 (2015)

22. Samanta, G.P.: Analysis of a nonautonomous HIV/AIDS model. Math. Model. Nat. Phenom. 5(6), 70-95 (2010)

23. Samanta, G.P.: Analysis of a nonautonomous HIV/AIDS epidemic model with distributed time delay. Math. Model. Anal. 15(3), 327-347 (2010)

24. Cai, L.M., Li, X., Ghosh, M., Guo, B.: Stability of an HIV/AIDS epidemic model with treatment. J. Comput. Appl. Math 229, 313-323 (2009)

25. Bera, S.P., Maiti, A., Samanta, G.P.: A delay dynamic model for HIV infected immune response. J. Appl. Math. Inform. 33, 559-578 (2015)

26. Hajii, M.A., Al-Mdallal, Q.: Numerical simulations of a delay model for immune system-tumor interaction. Sultan Qaboos Univ. J. Sci. 23(1), 19-31 (2018)

27. Dalal, N., Greenhalgh, D., Mao, X.: A stochastic model for internal HIV dynamics. J. Math. Anal. Appl. 341, 1084-1101 (2008)

28. Luo, Q., Mao, X:S Stochastic population dynamics under regime switching. J. Math. Anal. Appl. 334, 69-84 (2007)

29. Zhao, Y., Jiang, D., O'Regan, D.: The extinction and persistence of the stochastic SIS epidemic model with vaccination. Physica A 392, 4916-4927 (2013)

30. Nsuami, M.U., Witbooi, P.J.: Stochastic dynamics of an HIV/AIDS epidemic model with treatment. Quaest. Math. 42(5), 605-621 (2019)

31. Shulgin, B., Stone, L., Agur, Z:: Pulse vaccination strategy in the SIR epidemic model. Bull. Math. Biol. 60(6), 1123-1148 (1998)

32. Samanta, G., Bera, S.: Analysis of a chlamydia epidemic model with pulse vaccination strategy in a random environment. Nonlinear Anal., Model. Control 23(4), 457-474 (2018)

33. Agur, Z., Cojocaru, L., Mazor, G., Anderson, R.M., Danon, Y.L.: Pulse mass measles vaccination across age cohorts. Proc. Natl. Acad. Sci. USA 90, 11698-11702 (1993)

34. Pei, Y., Li, S., Li, C., Chen, S.: The effect of constant and pulse vaccination on an SIR epidemic model with infectious period. Appl. Math. Model. 35, 3866-3878 (2011)

35. Samanta, G.P.: Permanence and extinction of a nonautonomous HIV/AIDS epidemic model with distributed time delay. Nonlinear Anal., Real World Appl. 12(2), 1163-1177 (2011)

36. Zhu, W.Q., Cai, G.Q., Lin, Y.K.: On exact stationary solutions of stochastically perturbed Hamiltonian systems. Probab. Eng. Mech. 5, 84-87 (1990)

37. Li, D., Xu, W., Sun, C., Wang, L.: Stochastic fluctuation induced the competition between extinction and recurrence in a model of tumor growth. Phys. Lett. A 376, 1771-1776 (2012)

38. Diekmann, O., Heesterbeek, J.A.P., Metz, J.A.J.: On the definition and the computation of the basic reproduction ratio $R_{0}$ in models for infectious diseases in heterogeneous populations. J. Math. Biol. 28, 365-382 (1990)

39. Van den Driessche, P., Watmough, J.: Reproduction numbers and sub-threshold endemic equilibria for compartmental models of disease transmission. Math. Biosci. 180, 29-48 (2002)

40. Liu, M., Wang, K.: Persistence, extinction and global asymptotical stability of a non-autonomous predator-prey model with random perturbation. Appl. Math. Model. 36, 5344-5353 (2012)

41. Guan, Z.H., Hill, D., Shen, X.: On hybrid impulsive and switching systems and application to nonlinear control. IEEE Trans. Autom. Control 50, 1058-1062 (2005)

42. Gao, S., Chen, L., Nieto, J.J., Torres, A.: Analysis of a delayed epidemic model with pulse vaccination and saturation incidence. Vaccine 24, 6037-6045 (2006)

\section{Submit your manuscript to a SpringerOpen ${ }^{\mathcal{O}}$ journal and benefit from:}

- Convenient online submission

- Rigorous peer review

- Open access: articles freely available online

High visibility within the field

Retaining the copyright to your article

Submit your next manuscript at $\gg$ springeropen.com 